\title{
La posibilidad de la historia en la época de la responsabilidad. Vida, historia y ética en Hans Jonas
}

The possibility of history in the age of responsibility. Life, history, and ethics in Hans Jonas

\section{ERIC POMMIER}

Pontificia Universidad Católica de Chile

Instituto de Filosofía

8940000 Santiago, Chile

ericspommier@hotmail.fr

Abstract: This article shows that the Heideggerian concept of existence and historicity is confronted by an aporia that can be resolved by Jonas's trans-historical conception of life. Through this analysis it is also possible to show how the primary aim of the principle of responsibility is to maintain the possibility of history for humankind.

Keywords: Ethics, existence, history, life, responsibility.
Resumen: Se trata de mostrar aquí que la concepción heideggeriana de la existencia y de la historicidad encuentra una aporía que puede ser superada gracias a la concepción trans-histórica de la vida de Hans Jonas. Tal análisis es la condición para mostrar en qué sentido el principio de responsabilidad tiene como meta principal el cargo de mantener, para el hombre, la posibilidad por sí misma de la historia.

Palabras clave: Ética, existencia, historia, responsabilidad, vida. 
1 título de este articulo podría dejarnos bastante perplejos, ya que, primero, no vemos a priori por qué la historia debería ser una posibilidad y no un becho, y segundo, no parece haber razones fuertes para declarar que nuestra época es más una época de responsabilidad que otro periodo de la historia. Por último, no entendemos la relación que podría haber entre la cuestión de la historia y la de la responsabilidad, ya que la historia parece no tener nada que ver con la moral. Para disipar esta perplejidad es necesario recordar que la historia tiene un sentido ambiguo, a raíz de que esta palabra designa a la vez la "realidad" temporal del ser humano y el relato, tan verdadero como posible, de este desarrollo temporal individual y colectivo. Entonces, se plantea una primera pregunta: ¿Qué debe ser el ser humano para que tenga una historia y para que podamos interpretarla? ¿Cómo debemos entender al hombre de manera que, por una parte, podamos tener acceso a él como ser histórico y, por otra, a su historia? En otros términos:

a) ¿cómo podemos establecer la posibilidad ontológica de su conocimiento como ser temporal? ¿Qué es él ser de su temporalidad o cómo debemos entender la temporalidad del hombre?

b) y, ¿cuáles son las condiciones para entender la historia del ser humano? ¿Cómo es posible dar una inteligibilidad a hechos humanos que ya no son?

Efectivamente, la primera parte de esta pregunta plantea el problema agustiniano bien conocido del ser del tiempo: ¿si hay un pasado que ya no es, un presente que es, y un futuro que todavía no es, eso quiere decir que la realidad del tiempo humano toma refugio en el presente que solo es? ¿Pero, cuál puede ser el sentido del presente si no podemos relacionarlo con el pasado y el futuro a raíz de que ellos no son? Entonces, ¿Cómo establecer la realidad histórica del hombre para que podamos decir que él tiene una historia? ¿Sería necesario distinguir, como vamos a ver para resolver este problema, entre el ser del tiempo y su realidad? 
Además la segunda parte de nuestra pregunta — ¿cómo hacer reaparecer el pasado que ya no es? - plantea el tema de la verdad histórica. ¿En qué medida podemos tener la certeza de que la historia que contamos de un hombre, de un pueblo o de una nación sea verdadera si se trata de hacer vivir de nuevo hechos que ya no son? Sabemos que el historiador tiene una metodología propia para plantear este tipo de problema: él utiliza de manera crítica los archivos y los testimonios con el fin de restituir un vínculo de causalidad entre los eventos. Pero afirmar que el hombre del pasado puede ser entendido hace pensar que la humanidad tiene una estructura eterna, que ella cambia solamente de manera superficial, y, dicho en breve, que tiene una esencia eterna. Así, podemos entenderlo a pesar de la "distancia" del pasado. Pero esta hipótesis es contradictoria con el carácter histórico del hombre. No obstante, y al contrario, aceptar la historicidad "total" del hombre implica la relatividad de nuestro conocimiento y por lo tanto la imposibilidad de la ciencia histórica. Si el sentido de la vida del hombre cambia en cada época, entonces no hay otra opción que el escepticismo que es una descalificación de la verdad. ¿Podríamos hallar un tercer camino entre el esencialismo y el escepticismo respecto de la ciencia histórica? Vemos que la respuesta a esta doble pregunta es la condición esencial para afirmar la posibilidad ontológica de la ciencia histórica, gracias a la cual podemos decir que el hombre tiene una historia, y no solamente una historia individual y vivida, sino que también una historia contada y conocida. Así, se plantea el problema de la verdad del conocimiento histórico del hombre y de la relación entre la ontología —el ser histórico del ser humano- $\mathrm{y}$ la historia.

No obstante debemos enfocarnos sobre el segundo aspecto de nuestro cuestionamiento. De hecho, la ética del historiador consiste en separar el campo de la verdad y el del bien. Entender la historia no tiene nada que ver con actuar para su desarrollo moral o juzgar este desarrollo a partir de un patrón normativo. El historiador tiene una responsabilidad con respecto a la verdad y no así, frente al Bien. No se trata para él de contar una historia de bondades sino más bien, una verdadera historia; no de hacer el relato de lo que debe ser sino de los que es. Es claro que él puede integrar una referencia 
a los valores dentro de su análisis, puesto que los hombres pueden actuar tomando en cuenta valores, pero no debe entender el desarrollo histórico en relación con un ideal de bien. Sin embargo, no es verdaderamente posible desechar la reflexión sobre el bien cuando nos enfocamos en el tema de la historia. De hecho, hay una relación entre ética e historia. Primero, si le interesa la verdad al historiador, eso quiere decir que ella tiene un valor para él. Por lo tanto, estamos obligados a reconocer que la verdad de la historia humana tiene un valor ético: el interés del historiador no puede ser solamente el interés de un hombre egoísta en la medida que su interés se amplía hasta relatar y entender un fragmento de la historia del ser humano. Entonces debemos entender cuál podría ser la importancia para nosotros, la humanidad, de tal interés, y en qué medida el trabajo histórico puede sernos útil. Entender el curso efectivo de los eventos pasados puede ser un medio útil para orientar la finalidad de la historia según un curso más justo. Lo que quiere decir también que la definición del bien no puede evitar una reflexión histórica. La historia es el lugar donde se realizan o se estrellan las mejores intenciones, donde el bien puede pervertirse y donde el mal puede desarrollarse. Entonces no hay efectividad moral sin análisis de la historia. Desde este punto de vista, el tema de la historia no sólo remite al tema de la ontología sino que también al de la ética y, a la vez, el problema de la orientación ética de la historia. ¿Desde qué punto de vista se puede afirmar que debemos tener una orientación ética de la historia y cómo definir esta orientación de manera justa y realista?

Quisiera mostrar, en primer lugar, de qué manera Heidegger pretende resolver la aporía de la historia, entendida como ser del hombre y como ciencia, y en segundo lugar criticar el enfoque heideggeriano para mostrar, al alero de la filosofía de Hans Jonas, que esta concepción de la existencia no es ontológica sino abstracta e históricamente determinada, de modo que se necesita poner en primer plano la dimensión trans-histórica de la vida para entender la historicidad del hombre (y por lo tanto la posibilidad de una ciencia histórica). Por último, gracias a este análisis podremos indicar en qué sentido pertenecemos a la época de la responsabilidad, o dicho 
de otro modo, en qué medida el principio de responsabilidad que da una dirección a la historia tiene, por primera vez en la historia, como contenido el cargo de mantener, para el hombre, la posibilidad por sí misma de la historia. Ello presupone que el carácter histórico sería puesto en peligro por nuestra época, que es un periodo de peligro mayor y, en consecuencia, de mayor responsabilidad.

\section{Historia Y ONTOLOGÍA \\ a) La bistoricidad del hombre}

¿En qué sentido podemos afirmar que el ser humano es un ser histórico? La respuesta a esta pregunta es la condición ontológica de posibilidad de la ciencia histórica y de la afirmación de que el hombre puede tener una historia. Sin embargo, como ya hemos dicho, parece difícil identificar el ser del tiempo humano, puesto que se concentra en la realidad del instante presente, en tanto que el pasado y el futuro no son. Pero el momento presente tampoco tiene realidad, puesto que el sentido del presente supone una relación al futuro y al pasado. Si ellos no son, entonces el presente también parece ser ilusorio. No obstante Heidegger en Sein und Zeit, en particular en la sección 65, identifica el presupuesto sobre el cual descansa esta aporía. Tradicionalmente interpretamos la realidad de los instantes a partir de la realidad de la cosas, como si fueran idénticos a sí mismos, sin negatividad interior. La posición de una cosa es la exclusión de otra. Así el presente no es el pasado y no es el futuro que no son. Y es la razón por la cual no entendemos la relación entre los momentos del tiempo. El presente toma en él toda la realidad del tiempo a causa de su presencia. Entendemos la presencia del presente sobre el modelo de la presencia de la cosa, a partir de su permanencia. Como lo comenta Heidegger en Los problemas fundamentales de la fenomenología, refiriéndose a la aporía aristotélica del tiempo: "En esta pregunta por el ser del tiempo, Aristóteles comprende el ser en el sentido del ser subsistente. Si se toma el ser en este sentido, debemos decir entonces que el "ahora" ya no subsistente en el sentido de lo pasado y el ahora todavía no subsistente en el sentido de lo que 
viene no son, esto es, no subsisten. Visto así, en el tiempo, sólo el "ahora", que en todo "ahora" subsiste, es. Las aporías de Aristóteles respecto del ser del tiempo, que todavía hoy están vigentes, surgen de la identificación del concepto de ser con el de ser subsistente."1

Sin embargo podemos explicar por qué tenemos esta falsa relación al tiempo y también en qué consiste una concepción auténtica del tiempo, gracias a las nociones de angustia y de muerte. De hecho, la Grundstimmung de la angustia debe ser distinguida del miedo. El miedo es siempre miedo de algo a causa de algo. La angustia, al contrario, no tiene un objeto bien definido y no tiene una razón identificable. Mientras el miedo es una ruptura familiar de la familiaridad, la angustia es ruptura no-familiar de la familiaridad. No sabemos por qué estamos angustiados. Es que la angustia nos pone en presencia de la nada, nada desde el punto de vista de la realidad, del ser determinado u óntico, del ente. Quiere decir que descubrimos que lo que está en cuestión es nuestro propio modo de ser. Efectivamente, la apertura a sí mismo implica una relación a nuestra propia muerte. Con ella, descubrimos la posibilidad de la nada y, por lo tanto, la posibilidad como posibilidad. La relación con la muerte es la proyección de una posibilidad hacia la nada que existe como ente. Es la proyección de una posibilidad como posibilidad pura. La posibilidad de la nada devela el modo de ser de la posibilidad. La relación a la muerte es la condición de todo acontecimiento y también de nuestra auténtica historicidad. Con el descubrimiento del ser de lo posible, podemos entender la relación entre el pasado, el presente y el futuro: la presencia de un instante no significa la exclusión de otro. Por ejemplo el presente no significa el olvido del pasado. Efectivamente, existir significa ser una posibilidad y elegir una posibilidad tomando en cuenta nuestra muerte propia. En esta actitud, miramos el futuro respecto al cual el pasado y el presente adquieren un sentido. Por lo tanto, la presencia del futuro es compatible con la del pasado y del presente, ya que esta presencia no significa una permanencia substancial, como la de la cosa, sino más

1. M. Heidegger, Los problemas fundamentales de la fenomenología. Trad. Juan José Garcia Norro (Trotta, Madrid, 2000) 328. 
bien una presencia según el modo de ser de la posibilidad, del zu sein, a ser. El futuro no es desde el punto de vista de la cosa sino que es desde el punto de vista de la posibilidad, y presente y pasado también continúan existiendo como relación y en relación con nuestro proyecto futuro. Así podemos ver como Heidegger destaca la historicidad del Dasein y cómo éste da cuenta de la posibilidad del acontecimiento para el hombre. Entonces cuando nos entendemos según el modo de ser del ente, es debido a nuestra huida de nosotros mismos, como consecuencia de la angustia de la muerte. Preferimos entendernos falsamente como una cosa del mundo que, auténticamente, como un Dasein.

\section{b) La ciencia bistórica}

Además de ayudarnos a dar una inteligibilidad a la constitución ontológicamente histórica del hombre, esta concepción nos permite dar las condiciones de una comprensión del hombre de otra época y, por lo tanto, de la ciencia histórica. Sabemos las dificultades que se plantean para dar un fundamento a la ciencia histórica. Primero, y en relación con el objeto histórico, ¿cómo tener un acceso al pasado que ya no es? Segundo, y en relación con el sujeto que estudia la historia, ¿cómo evitar el riesgo de subjetivismo al interpretar los acontecimientos, al tomar en cuenta la idiosincrasia del historiador, las influencias que él sufre y las determinaciones de su propia época? Tercero, desde el punto de vista de la relación entre los acontecimientos, ¿debemos relacionarlos con la categoría de causalidad y poner en primer plano la existencia de leyes, con el riesgo de abolir la singularidad de estos acontecimientos, o dejarlos sin explicación al afirmar que las cosas se suceden y punto?

A decir verdad, el análisis heideggeriano permite superar estas dificultades, al tematizar el fenómeno de la relación del historiador con el campo histórico en lugar de enfocarse sobre el ideal abstracto del "objetivismo". Primero debemos decir que es posible hacer re-

2. Sobre la destrucción del ideal objetivista: S. Jollivet, Heidegger. Sens et histoire (1912-1927) (P.U.F., Paris, 2009) 106-114. 
vivir el pasado, como ya hemos visto, en la medida que el pasado no es una cosa determinada, bien definida que era y que ya no es. El pasado tiene un sentido solamente en relación con la proyección de un futuro. El pasado es vivido sólo cuando el historiador se hunde directamente en el mundo pasado, con respecto al cual las antigüedades tienen un sentido. Y, segundo, eso es posible porque el historiador es un Dasein que no solamente se interesa en una época sino que también es capaz de entenderla, relacionarse con el pasado, a causa de su modo de ser. De hecho, él es capaz de vivir el pasado según la modalidad de la posibilidad. Él puede relacionarse con una época, entendiendo lo que era su significación y sin abandonar su relación al presente. Desde este punto de vista, el debate entre objetivismo y relativismo parece abstracto. No hay otra opción para revivir el pasado que adoptar un punto de vista histórico, orientado hacia el futuro. La historicidad (die Geschichtlichkeit) del historiador no es un obstáculo para la comprensión de la historia, sino su única condición. Y no podríamos entender el pasado si tuviéramos un punto de vista objetivo, a-histórico sobre él. En consecuencia, no debemos interpretar esta falta de objetivismo como la señal de un relativismo del conocimiento de la historia. La comprensión histórica auténtica no es objetiva; ella echa una luz sobre una posibilidad de la existencia que ba sido. No se trata de reconstruir el pasado a partir del presente sino de revivir el pasado a la luz de una posibilidad de ser. De hecho la referencia a una común finitud permite traspasar las fronteras de las culturas y superar el relativismo. Debemos rechazar la alternativa entre una verdad a-histórica, abstracta y el relativismo. Es porque estamos, como Dasein, por delante de nosotros mismos, orientados por el futuro y tensados hacia el futuro, que es posible entender el proyecto de cada hombre, inclusive cuando se trata de un proyecto del pasado. No hay otra comprensión que una comprensión históricamente situada, a condición de entender esta situación según el modo de ser del proyecto, de la tensión hacia el futuro. Tercero, podemos rechazar también la alternativa de la ley universal y de la sucesión accidental de acontecimientos singulares. Es claro ahora que el sentido de la historia no tiene nada que ver, por una parte, con una explicación objetiva y causal supra-histórica 
$y$, por otra parte, con una continuación de acontecimientos que se ocurren y que desaparecen, ya que el pasado "se conserva" desde el punto de vista de una posibilidad que abre un futuro.

No podemos desarrollar este último punto, pero debo indicar que esta interpretación de la historicidad del ser humano y de la comprensión de la ciencia histórica supone liberarse de la tradición ontológica. Debemos destruir, lo que quiere decir adueñarse, a la tradición ontológica para hacer surgir nuevas posibilidades de comprensión y entender el ser no como ente sino como tiempo. Para entenderse propiamente, el Dasein debe no solamente entenderse desde el punto de vista del futuro existencial y no a partir de la presencia de la cosas dentro del mundo sino que también entender el entendimiento o la comprensión independientemente del legado de la tradición ontológica pasada, que comprende la comprensión como la operación de un sujeto que se representa un objeto. Para pensar la historicidad del Dasein y la condición ontológica de posibilidad del conocimiento histórico, a partir de la posibilidad y del futuro existencial, a partir de la apertura de un mundo, debemos romper con la tradición y pensar el Dasein, y también el pensamiento, a partir de la temporalidad existencial.

\section{VIDA E HISTORIA}

\section{a) Gnosticismo y existencialismo}

A primera vista, tenemos aquí una concepción muy convincente de la historicidad del hombre y que permite también entender concepciones del hombre de otra época. Por ejemplo, Hans Jonas va a apoyarse sobre la concepción del Dasein para echar una luz sobre la unicidad del movimiento gnóstico ${ }^{3}$ del fin de la Antigüedad tardía ${ }^{4}$.

3. Movimiento religioso de inspiración cristiana y neo-neoplatónica desarrollado entre el siglo I y III que afirma que el alma esta prisionera de un mundo malo creado por un dios malo. También el director de la tesis doctoral de Hans Jonas, Rudolf Bultmann, utilizará la analítica existencial para develar el sentido auténtico de la escatología bíblica.

4. Se puede consultar en particular: J. OliveIRA, Compreender Hans fonas (Editora Vozes, Petrópolis, 2014) 19-54. 
En lugar de constatar la pluralidad histórica de este movimiento, él va a mostrar que él se unifica a condición de entenderlo desde el punto de vista de un Dasein, arrojado-en-el-mundo. Para decirlo rápidamente, lo que es común entre la corriente gnóstica y la analítica del Dasein es el sentimiento de un divorcio entre la existencia y el mundo. Hay un sentimiento de extrañeza entre los dos, y el ser humano no se siente en su casa dentro del mundo. Es el caso con el concepto heideggeriano de Geworfenheit y también con el de alma gnóstica que se siente exiliada en este mundo. Todo pasa como si hubiera un dualismo entre el alma humana y el cuerpo biológico que tiene un equivalente respecto de la fractura entre el mundo y el hombre. De la misma manera que el hombre está separado del mundo, el alma es ontológicamente diferente del cuerpo, sin posibilidad de encarnarse en el mundo por medio de este cuerpo ${ }^{5}$. Además de esta primera analogía, Hans Jonas considera que podemos poner el acento sobre un segundo punto común. Tanto en el Gnosticismo como en la filosofía de Heidegger, hay una desvalorización del presente y una sobrevaloración del futuro. El alma perdida gnóstica debe fijarse en el futuro para anhelar reunirse con el principio olvidado. El Dasein debe proyectarse en el futuro auténticamente, tomando en consideración su muerte propia para dar sentido a su pasado. El presente es solamente un instante que tiene un valor en relación con este pasado y, sobre todo, al futuro. Él no permanece. No podemos tranquilizarnos en la contemplación de este presente.

Sin embargo, el éxito de la interpretación "existencialista” de la Gnosis va a conducir a Hans Jonas a una nueva interrogación. En efecto, el grado de afinidad entre los dos es inquietante y nos invita a hacer una pregunta. Él ha interpretado el movimiento gnóstico gracias a la concepción del Dasein. ¿Pero no podríamos interpretar la concepción existencialista a partir del modelo gnóstico? Como dice Jonas en The Phenomenon of Life:

5. Ver en particular: J. TAminiaux, "Les enjeux de la lecture gnostique de Sein und Zeit”, Etudes Phénoménologiques 33-34 (2001) 91-109. 
(...) las funciones interpretativas se invierten y devienen recíprocas: la cerradura se convierte en llave y la llave en cerradura. La lectura "existencialista" del gnosticismo, tan justificada como esta (...) por su éxito hermenéutico, nos invita, como a su contrapartida natural, a emprender el intento de una lectura "gnóstica" del existencialismo ${ }^{6}$.

Si esta afirmación es relevante, deberíamos concluir que la ontología fundamental de Heidegger no tiene una validez universal pero que la afinidad entre el existencialismo y la Gnosis se debe a una analogía a propósito de una situación histórica particular del ser humano. La clave aparentemente ontológica de la concepción existencialista del ser humano pertenecería en realidad a una situación histórica particular; o mejor dicho, la concepción ontológicamente histórica del Dasein, que sirve para entender al ser humano según su sentido propio, cualquiera sea su pertenencia a tal o cual periodo histórico, sería en realidad una concepción producida por el movimiento de la historia de las ideas. Como dice Hans Jonas: "Nuestra convicción de que la historicidad es algo esencial al ser humano como tal es también un producto histórico"7. Eso significaría que la interpretación ontológica del Dasein es en realidad histórica, que ella es el reflejo de una situación histórica bien determinada, y por lo tanto que ella no es una clave interpretativa universal. No podemos interpretar auténticamente todos los periodos históricos con ella. Así habría un relativismo histórico, ya que cada época tiene su propio sentido y las analogías entre ellas son excepcionales. Deberíamos concluir que la

6. H. JonAS, El principio vida: hacia una biología filosófica. Trad. J. Mardomingo (Trotta, Madrid, 2000) 281. La primera versión alemana es una traducción, en parte de Hans Jonas, de la versión inglesa The Phenomenon of Life (Northewestern University Press, Evanston, Illinois, 2001) y cuenta con algunas modificaciones. Su título es el de la primera versión inglesa no publicada de The Phenomenon of Life, es decir, Organism and Freedom: Organismus und Freibeit. Ansätze zu einer philosophischen Biologie (Suhrkamp, Frankfurt am Main, 1973). La nueva versión alemana póstuma de 1994 tiene otro título: Das Prinzip Leben, Ansätze zu einer philosophischen Biologie (Suhrkamp, Frankfurt am Main, 1994). La versión castellana El principio vida cit. es una traducción de este último libro.

7. H.Jonas, Pensar Sobre Dios y otros ensayos. Trad. A. Ackermann (Herder, Barcelona, 1998) 87. 
comprensión de la comprensión sería en última instancia el resultado del momento histórico, la consecuencia de la época y, por eso, no podríamos escapar del relativismo: cada época tiene su manera de entender el mundo y no podemos hallar algún punto común entre ellas. La comprensión pertenece al modo de ser del Dasein pero el Dasein heideggerriano pertenece a un momento de la historia análoga al del gnosticismo y no tiene un valor universal. O, para decirlo de otra manera, la idea que el hombre es ontológicamente histórico es un producto de la historia de las ideas. No es solamente la comprensión del ser humano la que es históricamente determinada y abstracta sino también la comprensión de la comprensión. Pensar al pensamiento como apertura al mundo, según la modalidad de la posibilidad temporalmente orientada, en función de un futuro mortal, sería un producto histórico que no tiene validez universal. Este juicio tendría al menos dos consecuencias, en primer lugar, sobre la antropología filosófica; en segundo lugar, sobre la ciencia histórica. Efectivamente, si la comprensión del ser humano es puramente histórica, entonces no podemos definir una esencia del hombre y, por lo tanto, parece imposible establecer una antropología.

Además, si no hay una esencia fija del hombre, no podemos identificar un sentido universal que sería la condición para entender al hombre a pesar de las variaciones históricas. Hemos visto que no era un problema desde el punto de vista heideggerriano, ya que podíamos entender lo histórico a partir de lo histórico ${ }^{8}$. Sin embargo, según el juicio de Jonas, eso significa que podríamos entender al otro desde el punto de vista del otro ${ }^{9}$, puesto que cada situación histórica modifica radicalmente la humanidad. Sin embargo, si no hay punto común entre ellos, si no hay una referencia trans-histórica no tenemos medio para evaluar una evolución histórica y también para entender un cambio. La ausencia de una antropología ontológica tiene como consecuencia la imposibilidad de la ciencia histórica.

Para resumir el nudo de este problema, podríamos considerar que la aporía es la siguiente. O bien afirmamos que hay una esencia

8. Ver 1.b.

9. H. Jonas, Pensar sobre Dios cit., 59 y 62. 
del hombre, y esa es la razón por la cual podemos afirmar la unidad del ser humano, entender las actividades humanas independientemente de la época histórica; pero no sólo parece difícil creer que el sentido de la humanidad es el mismo en cada época más también que las variaciones históricas no tienen una realidad fundamental. O bien reconocemos que el cambio histórico es permanente, que no hay punto fijo para dar cuenta del ser humano e interpretar los eventos del pasado, y por ello estamos obligados a concluir que no estamos seguros que tenga un ser humano esencial, que no hay inteligibilidad de la historia y que no podemos dar una dirección substancial a la historia "humana."

¿Existe una alternativa entre el historicismo escéptico y el esencialismo? Hemos visto que el intento de Heidegger conduce a la afirmación del relativismo, según Jonas. Sin embargo, Jonas trata de traspasar esta aporía y de sobrepasar la alternativa. Efectivamente, Jonas desvela la razón de la analogía entre el gnosticismo y la analítica de la existencia al mostrar que lo que es común es el olvido de la vida. Pero él desvela también la lógica de la historia de las ideas. En otros términos, podemos identificar una razón en la historia de la ontología cuyo motor es el olvido de la dimensión encarnada de nuestra existencia. La analogía entre el gnosticismo y la analítica del Dasein no es fortuita. Y gracias a esta comprensión histórica de la comprensión, él muestra que podemos identificar un elemento "trans-histórico", gracias al cual podremos escapar del relativismo de la comprensión histórica del hombre. Sería porque la filosofía olvida la vida, como es el caso del pensamiento heideggeriano, que ella sería conducida a desarrollar una interpretación abstracta e historicista de la existencia. Tomar en cuenta esta dimensión vivida hará posible escapar del historicismo sin, no obstante, caer de nuevo en el esencialismo, lo que va a obligar a Hans Jonas a hacer una lectura existencialista de la vida.

De hecho, la analogía entre el gnosticismo y el "existencialismo" de Heidegger indica a Jonas la posibilidad de una lógica de la historia orientada hacia el dualismo que rompe la unión ontológica entre el ser humano y el mundo. Puesto que el guión entre la existencia humana y el mundo es el cuerpo vivido, gracias al cual el 
alma puede encarnarse aquí y ahora, podemos sacar la conclusión de que esta lógica de la historia filosófica sería guiada por el olvido de la vida. Y así es. Jonas muestra que la historia del pensamiento del ser obedece a una "ontología de la muerte", según la cual la vida es solamente una lucha contra la muerte lo que explica que ella sea sencillamente sobrevivencia, sin apertura al exterior, consumo y apropiación. Ella no tiene un sentido transitivo, nada que tenga que ver con una percepción desinteresada que tiene un interés en la cosa por ella misma. De ahí la necesidad teórica de complementarla con una concepción del alma humana caracterizada como puro conocimiento ${ }^{10}$. Este dualismo aparece con el gnosticismo y culmina con la filosofía de Descartes pero se desarrolla en dos direcciones, materialista e "idealista" 1 . Efectivamente cada una permite resolver las contradicciones epistemológicas y ontológicas del dualismo pero con la condición de conservar este dualismo en segundo plano. Así, la materia del materialista es definida por su diferencia con el espíritu del dualismo, igual que el espíritu del idealismo con la materia ${ }^{12}$. Y la filosofía de Heidegger se inscribe plenamente en esta historia del pensamiento, pertenece a la familia del idealismo, a causa de la desencarnación del Dasein $^{13}$, y hereda su dualismo implícito ${ }^{14}$. Entonces no es una sorpresa ver que el sentido deriva de la pura actividad psíquica. Es así como esta manera de entender el sentido, como mero producto de la mente, implica su variabilidad en función de los símbolos dominantes del periodo histórico considerado.

10. R. BARBARAS, Introduction à une phénoménologie de la vie (Vrin, Paris, 2008) 136.

11. E. ЈАКОВ, Martin Heidegger und Hans fonas, Die Metaphysik der Subjektivität und die Krise der technologischen Zivilisation (Francke Verlag, Tübingen, 1996) 259-266

12. H. JoNAS, El principio vida cit., 31.

13. Sin embargo llama la atención el hecho que el joven Heidegger quería desarrollar una hermenéutica de la vida. Ver: S.-J. ArRIEN, L'inquiétude de la pensée: L’herméneutique de la vie du jeune Heidegger (1919-1923) (Épiméthée, Paris, 2014).

14. Aunque el proyecto de Sein und Zeit consista precisamente en romper con el dualismo substancial que caracteriza al ser humano. Sin embargo, ver R. BARBARAS, Introduction cit., 39-85. 


\section{b) Antropología de la vida e bistoria}

$\mathrm{Al}$ contrario, si entendemos que este historicismo es la consecuencia de una historia de la ontología que olvida la vida, debemos reintegrar la consideración de la vida en nuestra investigación ontológica, que es lo que justamente hace Hans Jonas. Sin entrar en los detalles de esta operación, debemos prestar atención al hecho de que la vida ya no puede ser concebida como una actividad de conservación de sí mismo, sino como una actividad de apertura al mundo ${ }^{15}$. La concepción existencialista de la vida permite traspasar el dualismo, al mostrar que el hombre representa un cierto grado de apertura al mundo. Como dice Hans Jonas:

Pero precisamente el cuerpo vivo, el organismo, presenta esa autotrascendencia en ambos sentidos (...). Hemos de describirlo como extenso e inerte, y a la vez como capaz de sentir y de querer, pero ninguna de las descripciones puede llegar a término sin traspasar el límite que la separa de la otra y sin prejuzgarla ${ }^{16}$.

La estructura metabólica del organismo consiste en un acto de autorenovación que tiene un sentido existencial en la medida en que el ser viviente despliega posibilidades para poder renovar sus componentes y para escapar de la muerte. Cambiar sin cesar es su ley y el principio de su "identidad”. Es así como él es condenado a abrir horizontes espaciales y temporales para identificar en su entorno lo que necesita. Es cierto que el organismo manifiesta un auténtico grado de libertad (a diferencia de la materia) puesto que su forma es independiente de su materia (que él debe renovar.) Pero esta libertad debe ser ejercida con necesidad para evitar la muerte. El organismo no puede conservar los mismos componentes. En tal caso sería un organismo muerto ${ }^{17}$.

15. Jonas elabora su ontología de la vida sin caer en una antropomorfización de esta vida. Ver C. FopPA, L'être humain dans la philosophie de la biologie de H. Fonas: quelques aspects, en G. HotTois, M.-G. PINSART (eds.), Hans Jonas. Nature et responsabilité (Vrin, Paris, 1993).

16. H. JONAS, El principio vida cit., 33 .

17. H. JonAs, El principio vida cit., 124-135. 
Concebir la vida según la "categoría" de la existencia, y la existencia humana a partir de la vida permite explicar, primero, cómo podemos entender el ser humano históricamente y, segundo, como una ciencia histórica es posible. En relación con el primer punto, debemos decir que la orientación de la existencia hacia el futuro proviene de su dimensión biológica. Como dice Hans Jonas:

La orientación interna a la siguiente fase de un ser que se tiene que prolongar a sí mismo da origen al tiempo biológico”, "el futuro es el horizonte temporal dominante que se abre ante el impacto de la vida, si es que el interés es el primer principio de su interioridad", "mientras que lo físico se halla enteramente determinado por lo que era (...), la vida siempre es ya también lo que será y lo que en ese momento se dispone a ser: en su caso, el orden extensivo del pasado y futuro está invertido desde el punto de vista intencional ${ }^{18}$.

El ser vivo quiere satisfacer su hambre, por lo tanto abre horizontes espaciales y temporales y por esta razón mira al futuro. La dirección temporal se explica a partir de las necesidades corporales y es la razón por la cual el futuro puede asegurar la unidad de los momentos del tiempo. Presente y pasado tienen sentido en relación con esta mirada al futuro y esta dirección tiene sentido en relación con el carácter vital del hombre. No podemos insistir aquí sobre este punto pero Hans Jonas define una escala de libertad dentro de la vida en función de su apertura al mundo, desde la planta hasta el hombre, pasando por el animal. Desde este punto de vista, la apertura al futuro es más libre con el hombre que con el animal, esto es, menos determinada por las necesidades vitales, gracias al poder simbólico del hombre que proviene de la apertura creciente de la vida hacia el mundo ${ }^{19}$.

\section{Ibidem, 134.}

19. R. RuBIO, Hans Jonas como teórico de la imagen. Análisis crítico de la recepción de fonas en el marco de la Bildwissenschaft, en E. Pommier y J.-C. GENs (eds.), Hans fonas. Número monográfico: "Alter" 22 (2014) 67-75. Ver también: M.-G. PINSART, Jonas et la liberté. Dimensions théologiques, ontologiques, éthiques et politiques (Vrin, Paris, 2002) 93-98. 


\section{c) La dimensión trans-histórica de la vida}

Pero, y es el segundo aspecto de nuestro análisis, la encarnación vital del hombre echa una luz sobre la posibilidad ontológica de la comprensión histórica. Para Jonas, el obstáculo para entender el pasado es doble. En primer lugar, se trata de entender al otro hombre, el hombre como otro, a pesar del solipsismo de la conciencia. Es el problema clásico de la filosofía cartesiana y de la intersubjetividad que el razonamiento por analogía no resuelve $\mathrm{e}^{20}$. En segundo lugar, se trata de entender el pasado a pesar de su alejamiento pero sobre todo a pesar de la univocidad lingüística del pasado. Al contrario del otro hombre contemporáneo con el cual podemos hacer un esfuerzo de comprensión gracias al diálogo y así disipar poco a poco, pero siempre parcialmente, la oscuridad, no podemos conversar con el hombre del pasado. Como dice Jonas:

La comprensión del presente tiene la ayuda del intercambio de afirmaciones; la comprensión de lo histórico solo dispone de la afirmación unilateral del pasado y por tanto, lo que crea la situación especial de la comprensión de la historia es el carácter absolutamente monológico de la comunicación histórica ${ }^{21}$.

Sin embargo, podemos reducir esta doble dificultad tomando en cuenta la encarnación del hombre. Cito a Jonas: “(...) conviene comenzar con lo biológico que presuponemos como evidente y que, pese a ser sub-histórico, impregna todo lo histórico"22. Jonas considera que existe un conocimiento intuitivo de la vida por la vida. Un animal puede entender el hambre de otro animal. En el caso del hombre también esta intuición existe pero se trata de coincidir con las posibilidades del otro hombre gracias a nuestras posibilidades de vida y no gracias a la efectividad de lo vivido. Como lo afirma Hans Jonas:

20. H. Jonas, Pensar sobre Dios cit., 66.

21. Ibidem, 74.

22. Ibidem, 76. Ver también: H. Jonas, Zwischen Nichts und Ewigkeit (Vandenhoeck \& Ruprecht, Göttingen, 1987) 3. 
El conocimiento de otro espíritu se basa, por tanto, en el fundamento de la humanidad compartida por todos los seres humanos. Pero ésta no obra por medio de la analogía, sino, al menos en la mayoría de los casos, por medio de la invocación y la evocación de las posibilidades que brotan de la naturaleza del ser humano y que se pueden despertar en ella. Son nuestras posibilidades las que nos permiten comprender y no necesariamente las precedencias efectivas de nuestra propia experiencia. De modo que comprendemos y respondemos en una medida mucho mayor con nuestro ser posible que con nuestro ser real ${ }^{23}$.

Y cuando se trata de comprender el pasado, también la importancia de "revivir" gracias a las posibilidades ofrecidas por la imaginación y su poder de empatía es esencial. Tal comprensión es posible gracias a nuestra capacidad de explorar al omni-humano, es decir las diferentes formas tomadas por la humanidad en el transcurso de la historia. Tenemos esta capacidad gracias al hecho de que, como hombres de la contemporaneidad, estamos avanzados desde el punto de vista de la historia, y porque la apertura gradual de la vida toma la forma del poder de imaginar y de proyectar posibilidades. Entonces, es porque la antropología de Jonas se apoya sobre una biología filosófica que él puede afirmar no solamente nuestro carácter ontológicamente histórico sino también nuestra capacidad de entender el pasado a partir del poder imaginativo. Podemos ser aún más precisos, ya que Jonas distingue tres horizontes abiertos por la propia vida del ser humano ${ }^{24}$. Así, él es capaz de entender tres tipos de significaciones fundamentales respecto de la vida del hombre del pasado. Primero, está el horizonte casi puramente biológico, es decir, la vida humana cuando ella utiliza las herramientas. Segundo, la fabricación de imágenes, que es aún más humana, anuncia la apertura en dirección de la verdad y del poder de la creatividad. Tercero, el hombre se interesa en la tumba, realiza sepelio. Este interés por la muerte es la señal de una reflexión sobre sí mismo, su destino y la ontología del

23. H. Jonas, Pensar sobre Dios cit., 71.

24. H. Jonas, Pensar sobre Dios cit., 76-78. 
ser en general. Vemos que lo que podríamos llamar la antropología de la vida, quiere decir la existencia de una antropología constituida a partir de una biología filosófica que es una ontología de la vida, permite explicar tanto el carácter ontológicamente histórico del hombre pero también la posibilidad de la ciencia histórica.

\section{HISTORIA Y ÉTICA DE LA RESPONSABILIDAD}

Una vez que fue establecida ontológicamente la historicidad del hombre y la posibilidad de entender la historia de la humanidad, podríamos preguntarnos para saber ¿por qué sería una buena cosa tratar de conocer esta historia? La posibilidad de entender nuestro pasado no significa que es bueno conocerlo. ¿En qué medida es posible afirmar que el conocimiento histórico tiene un valor? Al final del texto titulado Cambio y permanencia, Hans Jonas sugiere que es la condición para tener una historia propia. Lo cito:

Mientras nos encontremos en este movimiento del acontecer, para no ir ciegamente a la deriva, debemos tratar de comprender la historia, la nuestra y la de todo lo humano en esta Tierra. Si no lo hacemos, no tenemos derecho a nuestra propia historia, un derecho que aun así es bastante problemático ${ }^{25}$.

Eso significa que para no permanecer dependientes del pasado y de sus significaciones debemos entenderlo con el propósito de crear nuevas significaciones más libres. Por lo tanto, el conocimiento de la historia es la condición de una humanidad más libre. Sin embargo, me parece que para que la historia tenga este papel, para que la ciencia histórica sea vista como un poder liberador, debemos legitimar el valor de la historia, justificar el derecho a una historia propia. Esa es la razón por la cual después de haber afirmado que la ciencia histórica es la condición de la propia historia, la última palabra del texto ya citado pone el énfasis sobre la dificultad de legitimar este derecho a tal historia.

25. Ibidem, 88. 
No obstante, la filosofía de la historia ya nos ofrece un discurso dando las condiciones de una historia legítima. Immanuel Kant por ejemplo en Idea para una bistoria universal en clave cosmopolita muestra que debemos pensar el desarrollo histórico según la Idea de la humanidad. No se trata de constatar que la historia avanza en dirección de la justicia, pero de actuar como si fuera el caso, o de manera que la historia sea un progreso hasta el desarrollo de los derechos humanos. Podemos hallar en la historia ya vivida indicaciones de este progreso y tenemos el deber de hacer todo para agilizar este progreso. La historia tiene un valor a condición de ser un progreso moral y podríamos decir que el conocimiento de la historia es un medio para acercarse de este ideal.

Sin embargo, Hans Jonas, igual que Karl-Otto Apel después ${ }^{26}$, considera que Kant permanece ciego a las consecuencias reales del desarrollo humano y, particularmente a los efectos de su poder técnico, sobre la humanidad. Kant tiene una visión abstracta de la humanidad y subvalora el impacto real de su poder sobre la naturaleza y, en consecuencia, sobre la vida humana. Cito a Hans Jonas:

En general, toda capacidad "como tal” o "en sí" es buena, y sólo se vuelve mala por el abuso de ella. Por ejemplo, es innegablemente bueno poseer el poder de la palabra, pero malo para engañar a otros o llevarlos hacia su perdición. De ahí que sea plenamente sensato exigir: utiliza ese poder, auméntalo, pero no abuses de él. El presupuesto para ello es que la ética pueda distinguir claramente entre ambos usos, entre el uso correcto y el erróneo de una y la misma capacidad. Pero, ¿qué ocurre cuando nos movemos en un contexto en el que cualquier uso de la capacidad a gran escala, por muy buena que sea la intención con que se acomete, lleva consigo una orientación con efectos crecientes en última instancia malos, que están in-

26. K.-O. APEL, Diskurs und Verantwortung. Das Problem des Übergangs zur postkonventionellen Moral, (Suhrkamp, Frankfurt am Main, 1988) y K.-O. APEL, Die ökologische Krise als Herausforderung für die Diskursethik, en D. BöHLER (ed.), Ethik für die Zukunft. Im Diskurs mit Hans Fonas (C. H . Beck, Munich, 1994) 369404. 
separablemente unidos a los "buenos" efectos perseguidos y al alcance de la mano y al final quizá los superen en mucho? Si éste fuera el caso de la técnica moderna-como suponemos por buenas razones-, entonces el tema del uso moral o inmoral de sus poderes ya no es una cuestión de distinciones cualitativas evidentes por sí mismas y ni siquiera de intenciones, sino que se pierde en un laberinto de suposiciones cuantitativas sobre consecuencias últimas y tiene que hacer depender su respuesta de su aproximación. La dificultad es que no sólo cuando se abusa de la técnica con mala voluntad, es decir, para malos fines, sino incluso cuando se emplea de buena voluntad para sus fines propios altamente legítimos, tiene un lado amenazador que podría tener la última palabra a largo plazo. Y el largo plazo está de algún modo inserto en la acción técnica ${ }^{27}$.

Al afirmar una moral de la intención, él deja de lado la evaluación de las malas consecuencias de buenas acciones desde el punto de vista de la intención. El buen padre de familia toma su coche cada mañana para acompañar a sus hijos a la escuela y para ir al trabajo, lo que es bueno en términos de una moral de la intención, pero al actuar así él agiliza el desarrollo de la polución que tiene malas consecuencias sobre el planeta y la vida humana. Entonces el ideal de la justicia y de los derechos humanos no parece ser suficiente para orientar el desarrollo de la historia. Necesitamos una ética de la responsabilidad que tome en cuenta las consecuencias del desarrollo humano sobre la naturaleza y la humanidad.

La argumentación jonasiana enfatiza el riesgo del desarrollo técnico excesivo que podría poner en peligro la posibilidad misma de la humanidad de tener una historia. Hemos dicho que la filosofía del progreso moral destacada por Kant podría ser insuficiente para limitar los excesos de la técnica. Pero deberíamos añadir que esta ideología podría, en una cierta medida, esconder y también facilitar estos excesos. Efectivamente para Jonas la filosofía de Kant es el

27. H. Jonas, Técnica, medicina y ética. Trad. C. Fortea Gil (Paidos, Barcelona, 1997) 33-34. 
primer paso antes de la inmanentización del Ideal. Él confía a la historia el cargo de realizar el ideal. Hegel, y Marx después, va a encargar al proceso histórico la responsabilidad de realizar el fin ideal, según Hans Jonas. Como lo afirma:

Lo que en el esquema platónico se confiaba al progreso personal del individuo, aquí se encomienda o se exige al decurso externo de la historia; y la participación del individuo en el conjunto del proceso no puede ser sino un fragmento más como en todos los modelos de "progreso"-. Ciertamente Kant no podía aun conceder que el proceso histórico fuera una vía suficiente para alcanzar el ideal. Pues para él el tiempo no es propiamente real, pertenece sólo al mundo fenoménico, y de su causalidad no cabe aguardar que aporte nunca como estado general la coincidencia de felicidad y dignidad moral que el "bien supremo" exige (...) Fue Hegel quien dio el paso definitivo a la inmanentización radical. La idea reguladora se convierte, por encima de los que desean y actúan, en idea constitutiva; y el tiempo, que en modo alguno es ya mero fenómeno se convierte en el medio autentico de su realización, que acontece gracias al automovimiento de la idea. (...) Marx llevó a cabo la famosa inversión del dinamismo hegeliano colocándolo "sobre los pies" (...) [el marxismo] en la medida en que cree conocer el rumbo y la meta, es todavía heredero de la idea reguladora kantiana, pero la despoja de su infinitud y la traslada a lo finito $(\ldots)^{28}$.

Sin entrar en los detalles de tales afirmaciones, podemos decir que la secularización del mundo ideal transforma el ideal en una utopía a juicio del filósofo. Mientras la concepción kantiana del ideal implicaba una distancia entre el ideal y la realidad, la filosofía después de Kant va a desarrollar una lógica utópica, quiere decir una identidad entre la efectividad y el valor absoluto. La filosofía del ideal permitía condenar las utopías que afirman que la realidad debe concretizar

28. H. Jonas, El principio responsabilidad. Trad. A. Sánchez Pascual (Herder, Barcelona, 1995) 210-211. 
completamente el ideal ya que, desde el punto de vista de la Idea, jamás la historia se conforma a tal Ideal. Sin embargo, esta filosofía se vuelve una filosófica de la utopía, de la que Ernst Bloch con Das Prinzip Hoffnung ${ }^{29}$ es el mejor representante, a causa de la inmanentización completa del ideal. De hecho, en lugar de considerar la naturaleza como un obstáculo que debemos superar gracias a la razón, la razón se vuelve en el mejor medio para transformar esta naturaleza con el fin de darnos la esencia que necesitamos en función del objetivo ideal. Eso quiere decir que la razón moral se transforma en razón técnica. En lugar de utilizar la técnica como un primer paso para alejarse de la naturaleza y aproximarse al ideal moral de la razón práctica, la técnica se vuelve "moral” por sí misma. Detrás de la escatología aparente de la filosofía utópica, Hans Jonas diagnostica un movimiento no escatológico que es el movimiento del desarrollo técnico autónomo que actúa "como" un destino. Como él dice:

La crítica de la utopía, a la que con lo dicho hemos puesto fin, hubiera resultado excesivamente prolija si el utopismo marxista, en su estrecha alianza con la técnica, no representara una versión "escatológicamente" radicalizada de aquello hacia lo cual ya se encuentra en camino, de modo nada escatológico, bajo el sino del progreso, el universal ímpetu escatológico ${ }^{30}$.

Entonces el progreso, que da un valor al futuro y que invita a construir un futuro mejor que el presente, el progreso puesto en primer plano por la lógica escatológica significa en realidad una destrucción de la historicidad del hombre, ya que el movimiento técnico que es subyacente a esta lógica nos invita a un movimiento, quedando en el mismo lugar. Cuando creemos que es el bueno que debe dar la dirección del futuro, en realidad es la lógica técnica que actúa. Aparentemente el progreso mira al futuro pero en realidad quedamos prisioneros de un presente que se repite y que elimina toda

29. E. Bloch, Das Prinzip Hoffnung (Suhrkamp Verlag Gmbh, Frankfurt am Main, 2013.)

30. H. JONAS, El principio cit., 354. 
posibilidad de novedad ${ }^{31}$. De hecho, al transformar la naturaleza y la vida, en la cual hay una apertura al mundo, gracias a la cual podemos darnos cuenta del modo de ser que unifica los diferentes momentos del tiempo, como ya hemos visto, y que pone el énfasis sobre el futuro, la técnica destruye el modo de ser de la posibilidad y del futuro, transforma la vida en una cosa "real", en un ente que podemos modificar, refabricar. Por ejemplo, con la clonación fijamos una característica ventajosa para sustraerla al azar. Así, se destruye el surgimiento de la novedad ${ }^{32}$. Es el reino de la identidad. Para tomar otro ejemplo, podríamos creer que la ingeniería genética fabrica nuevas posibilidades de vida, pero en realidad lo que es fabricado es un tipo genético previsto y creado en función de su interés social o individual. Y eso es en desmedro de la aparición de la pura nove$\mathrm{dad}^{33}$. Entonces lo que debemos ver es que la técnica abole el futuro en beneficio de un presente ya conocido, lo que sería el equivalente de una destrucción del mundo.

Tenemos aquí la razón por la cual Jonas considera que una ética de la responsabilidad es necesaria. El papel de esta ética es de conservar la posibilidad de tener una historia para el hombre. La conservación de la vida y de la naturaleza es la condición de la preservación de la historicidad del hombre y de su libertad. Desde este punto de vista, me parece que estamos claramente en la época de la responsabilidad. Por primera vez, el hombre tiene, gracias a su poder, la posibilidad de destruir su mundo. No significa que el mundo material va a explotar o que el hombre, del punto de vista de la ciencia biológica, va a desaparecer, pero quiere decir que la posibilidad como posibilidad está en peligro, que entramos en una época en la cual es posible que ya no tenga posibilidad. El poder de la técnica y la amenaza que él representa desvela una dimensión esencial de

31. Es también lo que hace valer el gran filósofo checo Jan Patočka. Ver: J. PATOčKA, Libertad y Sacrificio. Trad. I. Ortega Rodríguez (Salamanca, Sígueme, 2007) 235.

32. Ver en particular: H. JonAs, Técnica cit., 109-134.

33. Para la discusión ética de otro tipo de biotecnológica, la de la extensión temporal de la vida humana gracias a la regeneración bioquímica de las células, ver: L. VoGEL, Is Ageing a Gift? Bioconservatism and the Ethics of Gratitude, en J.-S. Gordon, H. BurcKharT (eds.), Global Ethics and Moral Responsibility, Hans fonas and his Critics (Ashgate, Dorchester, 2014). 
nuestra vida: nuestro deber de protegerla como lugar de los posibles. $\mathrm{Al}$ mismo tiempo que descubramos los peligros de la técnica, aprendemos nuestro deber de responsabilidad. Como él dice: "solamente sabemos qué está en juego cuando sabemos que está en juego" ${ }^{34}$. Entendemos que por primera vez en la historia humana, la posibilidad de la historia está en peligro a causa del poder humano.

Sin embargo podríamos creer que esta defensa de la vida, como condición de la historicidad del hombre, va a conducirnos a dejar de lado la exigencia de justicia. Karl-Otto Apel, por ejemplo, reprocha a Jonas sacrificar el imperativo de dignidad y de justicia, que define la humanidad como humanidad, en beneficio de la preservación de la humanidad como especie. Si la técnica pone en peligro la vida humana a causa de las poluciones en el ambiente, entonces podría ser útil reducir la población mundial para disminuir el nivel de consumo y las poluciones. Podríamos imaginar una cosa tan inhumana y cruel como dejar morir el continente africano en el nombre de la sobrevivencia de la humanidad en general, si el valor de justicia, de igualdad humana es descartado en beneficio de la responsabilidad para la sencilla vida de la especie humana. No obstante, es claro que el imperativo de responsabilidad de Jonas que manda preservar la vida y la vida humana en particular no abandona la exigencia de justicia. Efectivamente, la responsabilidad por la vida humana es responsabilidad por la humanidad como bumanidad, como capaz de abrir un mundo de posibilidades, como libre. Entonces la vida humana no sería preservada si debiéramos violar la libertad humana ${ }^{35}$. Para decirlo brevemente, no sólo el principio responsabilidad es compatible con la justicia, sino que él es también una invitación a renovar este principio y a tomar en cuenta la posibilidad de compartir el peso social de las reformas necesarias en función de la riqueza de cada país, en función de su estado de desarrollo. Los países ricos deberían pagar más para ayudar a los países que están desarrollándose y estos países deberían aceptar no tener el mismo tipo de desarrollo que el

\section{Ibidem, 65.}

35. E. Pommier, La responsabilité en discussion. Apel-fonas, "Revue Philosophique de la France et de l'Etranger" 137/4 (2012) 495-514. 
que han conocido los países ricos, y que fue tan malo desde el punto de vista ambiental. Es así como el principio de responsabilidad en la justicia es lo que necesitamos para mantener nuestro derecho a una historia abierta a un futuro para la humanidad.

Para concluir, hemos visto que la historicidad del hombre se justifica a partir de su vida entendida como existencial. Hemos visto también que la ciencia histórica es posible al tomar en cuenta nuestro poder de simpatizar con el hombre del pasado desde el punto de vista de nuestra apertura amplia y variada al mundo. Por fin, hemos tratado de mostrar que el conocimiento de la historia y que el esfuerzo por tener una historia propia implicaba aplicar un principio de responsabilidad bastante nuevo que permita protegernos de los excesos de la técnica. 